(с) Л.Я. Рожинская, Ж.Е. Белая, А.С. Луценко

ФГБУ Эндокринологический научный центр Минздрава России, Москва, Российская Федерация

Обзор литературы посвящен проблеме лечения вторичного гиперпаратиреоза (ВГПТ) у пациентов с терминальной стадией ХБП на гемодиализе. В основе патогенеза ВГПТ лежит депривация D-гормона с запуском патофизиологических механизмов нарушения костного ремоделирования, повышения ФРФ-23, ПТГ, изменениями содержания фосфора и кальция в сыворотке крови, нарушениями в чувствительности и регуляции кальций-чувствительного рецептора (КЧР), что в последующем приводит к значимым изменениям структуры костной ткани и кардиоваскулярным осложнениям. В списке препаратов, применяемых для лечения ВГПТ у диализных больных, значимое место занимают агонисты КчР, до недавнего времени представленных единственным препаратом для приема внутрь - цинакальцетом с убедительно доказанной эффективностью. В настоящее время в США, Европе и России зарегистрирован новый кальцимиметик для внутривенного введения 3 раза в неделю - этелкальцетид. В обзоре представлены результаты клинических исследований этого препарата, показавшего преимущества в эффективности в сравнении с цинакальцетом и аналогичный профиль по побочным эффектам. Предполагается повышение приверженности к лечению ВГПТ у диализных пациентов за счет снижения кратности введения препарата и возможности его введения в диализных центрах.

кЛЮчЕВЫЕ СЛОВА: Вторичный гиперпаратиреоз; хроническая болезнь почек; гемодиализ; кальцимиметики; этелкальцетид; цинакальцет;

\title{
NOVEL TREATMENT OPTIONS FOR SECONDARY HYPERPARATHYROIDISM IN END-STAGE KIDNEY DISEASE PATIENTS ON HEMODIALYSIS THERAPY
}

(c) Liudmila Y. Rozhinskaya, Zhanna E. Belaya, Alexander S. Lutsenko

Endocrinology Research Centre, Moscow, Russian Federation

Pathogenesis of secondary hyperparathyroidism is based on D-hormone deprivation, leading to bone remodeling impairment, increase in FGF-23, PTH levels, changes in blood calcium and phosphorus levels. Taken together with alteration of calcium-sensing receptor (CaSR) sensitivity, these changes result in alteration of bone structure and cardiovascular complications. CaSR agonists are one of the most important medications for treatment of secondary hyperparathyroidism in dialysis patients. Until recently, there was only one CaSR agonist with proven effectiveness - cinacalcet, which is administered per os, daily. Now, a new drug is registered in US, Europe and Russia - etelcalcetide, which is administered intravenously 3 times a week. In this review we focus on results of clinical trials regarding etelcalcetide effectiveness and possible compliance benefits.

KEYWORDS: Secondary hyperparathyroidism; chronic kidney failure; hemodialysis; calcimimetic agents;

\section{ВВЕДЕНИЕ}

Под вторичным гиперпаратиреозом (ВГПТ) понимается избыточная секреция паратгормона (ПТГ) в ответ на хроническую гипокальциемию. Наиболее тяжелые формы стойкого вторичного гиперпаратиреоза развиваются у пациентов с терминальной почечной недостаточностью [1].

По данным «Обзора общественного здравоохранения и исследования питания NHANES», среди населения США распространенность терминальной хронической болезни почек (ТХБП) с 1992 по 2002 г. увеличилась с 0,6 до 1,1\% [2]. Результаты скрининговых исследований свидетельствуют о том, что частота выявления отдельных стадий ХБП примерно сопоставима вне зависимости от страны и популяции и около 10-17 \% взрослого населения страдает ХБП. При этом диагностирована болезнь лишь у 1\% населения. По последним данным ВГПТ составляет 20 - 56\% всех нарушений фосфорно- кальциевого обмена при ХБП [3].

\section{ПАТОГЕНЕЗ РАЗВИТИЯ ВТОРИЧНОГО ГИПЕРПАРАТИРЕОЗА ПРИ ХРОНИЧЕСКОЙ БОЛЕЗНИ ПОЧЕК}

Уменьшение массы действующих нефронов при хронической почечной недостаточности (ХПН) ведет к гиперфосфатемии, сопровождающейся реципрокным снижением ионизированного кальция в крови. Гипокальциемия и гиперфосфатемия стимулируют синтез ПтГ околощитовидными железами. С гиперфосфатемией связывают возникновение резистентности скелета к кальциемическому действию ПТГ, что приводит к нарушению костного обмена и усугубляет гипокальциемию. Кальций воздействует на процессы синтеза ПтГ через кальций-чувствительные рецепторы, представленные в околощитовидных железах (ОЩЖ), количество и чувствительность к внеклеточному кальцию которых при уремии также уменьшаются. При нарастании ХПН возникает дефицит синтезируемого 
в почках кальцитриола, а также уменьшается число рецепторов к кальцитриолу в ОЩЖ, в результате ослабевает супрессивный эффект кальцитриола на синтез и секрецию ПТГ [4].

Недавно полученные данные указывают, что снижение 1а-гидроксилирования кальцидола происходит не только по причине атрофии почечной паренхимы, но и из-за резкого увеличения уровня фактора роста фибробластов-23 (ФРФ-23), являющегося ключевым регулятором обмена фосфора в организме. ФРФ-23 синтезируется остеоцитами (клетками костной ткани) и обладает выраженным фосфатурическим действием за счет подавления реабсорбции фосфата в проксимальных почечных канальцах. Кроме того, ФРФ-23 значительно подавляет активацию витамин D и переводит его в неактивную форму путём увеличения активности гидроксилирования в положении 25 [5]. ФРФ-23 также рассматривается как стимулятор секреции ПТГ, что создает новый гормональный каскад, защищающий организм от гиперфосфатемии на ранних стадиях ХБП [6]. На преддиализных стадиях даже значительное увеличение ФРФ-23 не способно предотвращать развитие гиперфосфатемии и формирования ВГПТ [7]. Данные проспективных исследований свидетельствуют о том, что повышение уровня ФРФ-23 ассоциируется со смертностью пациентов и сосудистой кальцификацией, независимо от установленных факторов риска [8]. У пациентов на диализе после паратиреоидэктомии уровень ФРФ-23 медленно снижается, однако нормализации его уровня не наблюдается. После почечной трансплантации также не происходит полной нормализации уровня ФРФ-23, что объясняет случаи гипофосфатемии. Для связи с рецептором и реализации гормонального эффекта ФРФ-23 нуждается в присутствии корецептора - белка Klotho, который в норме синтезируется в почечной ткани. Кофактор ФРФ-23 - белок Klotho продуцируется и секретируется клетками проксимальных почечных канальцев и клетками ОЩЖ. В экспериментальном исследовании установлено, что трансмембранная форма белка Klotho является корецептором для ФРФ-23 и участвует в регуляции обмена фосфора, кальция и витамина D [5]. Экспрессия белка Klotho снижена у больных с ХБП, на основании чего предполагают, что данный белок является ренопротективным фактором. Поскольку повышенный уровень сывороточного ФРФ-23 у больных с ХБП предшествует гиперфосфатемии, резистентность к ФРФ-23 может быть одним из наиболее ранних проявлений нарушения метаболизма фосфора при ХБП [6]. Предполагают, что развитие резистентности к ФРФ-23 вызвано снижением почечной экспрессии Klotho. В связи с этим низкий уровень экспрессии Klotho в почках может быть фактором неблагоприятного отдаленного прогноза у диализных больных [8]. Все вышеуказанные процессы приводят к возникновению характерных для ВГПТ костно-минеральных нарушений (КМH), клинически проявляющихся с одной стороны различными поражениями костей, вплоть до фиброзного остеита, остеомаляции и переломов, с другой стороны - кальцификацией стенок сосудов, клапанов сердца, что приводит к высокой сердечно-сосудистой заболеваемости и смертности. КМН могут сопровождаться миопатией, нейропатией, зудом, кожными проявлениями, связанными с эктопической кальцификацией и кальцифилаксией, а также усугублять нефрогенную анемию $[9,10]$.

\section{ЛЕЧЕНИЕ НАРУШЕНИЙ КАЛЬЦИЙ-ФОСФОРНОГО ОБМЕНА ПРИ ВГПТ И ТХБП}

В настоящее время используются различные группы препаратов, направленных на коррекцию гипокальциемии, гиперфосфатемии и снижение ПТГ, что в конечном итоге должно предотвратить или замедлить развитие фатальных осложнений. Для этих целей применяются кальцийсодержащие и не содержащие кальций фосфатбиндеры; активные метаболиты витамина D и их аналоги (кальцитриол, альфакальцидол, парикальцитол) которые как правило эффективны при легких и умеренно выраженных формах ВГПТ, так как с прогрессированием заболевания и гиперплазией околощитовидных желез возникает резистентность к лечению и усугубляются побочные эффекты терапии. Существенным прорывом в понимании патогенеза, и затем в лечении ВГПТ, было открытие в 1993 г. EM Brown и соавт. кальций-чувствительных рецепторов (КЧР), локализованных в том числе и в ОЩЖ, и как следствие - понимание их роли и нарушения их регуляции при ВГПТ, а также разработка эффективных кальцимиметиков для лечения ВГПТ при ХБП [11-13].

В зависимости от сложившейся ситуации, можно рассмотреть различные варианты нарушений минерального обмена и костного ремоделирования. Основные схемы действия клинициста $[4,14]$ при различных нарушениях кальция и фосфора при ВГПТ суммированы в таблице 1.

Исходя из приведенной таблицы и накопленного клинического опыта, при умеренном и выраженном гиперпаратиреозе, за исключением ситуаций, связанных с гипокальциемей, современное и успешное медикаментозное лечение ВГПТ невозможно без применения агониста КЧР (кальцимиметика) - цинакальцета (ЦКТ). КЧР - G-протеиновый рецептор, экспрессируемый на поверхности главных клеток ОЩЖ, тонко настроенный на незначительные колебания уровня кальция в крови. Главная функция КЧР в ОЩЖ - регуляция секреции ПТГ путем поддержания в крови и костной ткани минерального гомеостаза. Активация КЧР нормальным физиологическим уровнем кальция замедляет продукцию и секрецию ПТГ, в то время как гипокальциемия ведет к уменьшению активности КЧР и увеличению ПТГ. [11]

Одной из терапевтических возможностей лечения ВГПТ явилось создание препарата, мишенью которого является активация КЧР и снижение продукции ПТГ, первый из таких препаратов - цинакальцет. Концептуально лекарство, которое влияет на КЧР и может либо понижать порог рецепторной активации в ответ на флюктуации внеклеточного кальция, либо прямо активировать рецептор, связываясь с внеклеточным доменом. Цинакальцет действует как аллостерический модулятор КЧР, потенцирующий действие внеклеточного кальция, понижая порог рецепторной активации, что ведет к снижению секреции ПТГ [15].

К настоящему времени накоплено значительное количество исследований и клинического опыта, доказывающих эффективность цинакальцета в отношении снижения ПТГ, кальция и фосфора и даже FGF23 - как суррогатных критериев эффективности лечения ВГПт [16-18]. В крупных клинических исследованиях доказана способность препарата снижать частоту переломов бедренной кости на 54\% у диализных пациентов, в сравнении с группой плацебо на 92 \% реже применять паратиреоидэктомию и снижать 


\section{Фосфор выше желаемого значения (рефе- рентного интервала)}

Фосфор в рам-

ках желаемого значения

\begin{tabular}{|c|c|}
\hline исимо & Й ди \\
\hline $\begin{array}{l}\text { от уровня } \\
\text { кальция }\end{array}$ & $\begin{array}{l}\text { Советы по диете по уменьшению содержания } \\
\text { фосфора }\end{array}$ \\
\hline
\end{tabular}

Отменить добавки карбоната кальция. Снизить дозу или отменить активные метабо*Высокий уровень кальция в сыворотке крови литы витамина D/аналоги витамина D (альфакальцидол, кальцитриол, парикальцитол). Назначить фосфатбиндеры: севеламера гидрохлорид, лантана карбонат..

Если уровень паратгормона высокий (более 300-500 нг/мл) - инициация или увеличение дозы цинакальцета гидрохлорида.

Прием карбоната кальция с каждой едой или немедленно после нее (не более 1500 мг). Назначить или увеличить дозу не кальцийНормальный содержащих фосфатбиндеров: севеламера уровень каль- гидрохлорида, лантана карбонат) Уменьшить ция в сыво- дозу или отменить аналоги (парикальцитол/ ротке крови активных метаболитов витамина D).

Если ПТГ высокий - необходимо инициировать или увеличить дозу цинакальцета гидрохлорида

Увеличить дозу или назначить карбонат каль${ }^{*}$ Сниженный кальций в сыворотке крови ция. Назначить некальций-содержащие фосфатбиндеры: севеламера гидрохлорид, лантана карбонат).

Уменьшить дозу или отменить цинакальцета гидрохлорид

\section{Фосфор ниже желаемого уровня}

Убедитесь, что пациент адекватно питается, исключить тяжелый дефицит питания

Отменить добавки карбоната кальция Снизить дозу или отменить Уменьшить дозу или отменить аналоги/активные карбонат кальция, уменьшить дозу метаболиты витами- или отменить некальций-содержана D.

Если уровень щие фосфатбиндеры (севеламера паратгормона вы- Уменьшить дозу или отменить аналосокий - инициация ги витамина D/активные метаболиты или увеличение дозы цинакальцета гидрохлорида.

Уменьшить дозу или отменить кальций карбонат, некальций-содержащие фосфатбиндеры.

Назначить или увеличить дозу активных метаболитов/аналогов витамина D

Уменьшить дозу или отменить кальций-несодержащие фосфатбиндеры: севеламера гидрохлорид, лантана карбонат Увеличить дозу активных метаболитов витамина D и/или добавить карбонат кальция между приемами пищи.

Уменьшить дозу или отменить цинакальцета гидрохлорид

*Высокий и низкий уровень кальция в сыворотке крови может также контролироваться изменением состава диализата. В ряде случаев может потребоваться хирургическое лечение вторичного гиперпаратиреоза, если повышенный уровень паратгормона, кальция и фосфора не удается компенсировать медикаментозными методами лечения.

число госпитализаций, связанное с сердечно-сосудистой заболеваемостью на 39\% [17, 19, 20].

Тем не менее, особенно при выраженном ВГПТ существует проблема резистентности и к цинакальцету, также его применение ограничено такими побочными эффектами, как тошнота, рвота, диарея, гипокальциемия, необходимостью тщательно титровать дозу и принимать препарат ежедневно 1-2 раза в день, что существенно снижает комплаентность пациентов и способность к длительному его приему $[21,22]$. Это послужило стимулом к дальнейшему поиску кальцимиметиков с возможностью решения ряда указанных проблем.

Этелкальцетид (ЭКЦ) - новый кальцимиметик, синтетический октапептид, включающий 7-D аминокислот, вместо обычных L-аминокислот. Он представляет собой дисульфид $\mathrm{N}$-ацетил-D-аргинамид, связанный с L-цистеином и функ- ционирует как активатор КЧР. Этелкальцетид взаимодействует с КЧР в местах, отличных от цинакальцета. Хотя острый фармакодинамический эффект этелкальцитида схож с таковым цинакалцета, фармакокинетический профиль их различен [23]. В таблице 2 суммированы основные свойства цинакалцета [24] и этелькальцетида [23, 25, 26].

К настоящему времени проведено 2 крупных международных многоцентровых исследования эффективности применения этелкальцетида у пациентов с ВГПТ и ТХБП на гемодиализе $[9,10]$ и одно - японское двойное слепое плацебо-контролируемое многоцентровое исследование [27].

Международные многоцентровые регистрационные исследования были запланированы одновременно и проводились параллельно. Первое исследование рандомизированное, двойное, слепое, плацебо-контро- 
Таблица 2. Основные свойства эинакальцета и этелкальцетида

\begin{tabular}{|c|c|c|c|c|c|}
\hline Показатель & \multicolumn{2}{|l|}{ Цинакальцет } & \multicolumn{3}{|c|}{ Этелкальцетид } \\
\hline Класс & \multicolumn{2}{|l|}{ Кальцимиметик } & \multicolumn{3}{|l|}{ Кальцимиметик } \\
\hline Описание & \multicolumn{2}{|c|}{$\begin{array}{l}\text { Низкомолекулярное органическое соедине- } \\
\text { ние }\end{array}$} & \multicolumn{3}{|c|}{$\begin{array}{l}\text { Синтетический агонист, содержащий } 7 \text { амино- } \\
\text { кислотных остатков и связанный с L-цистеином }\end{array}$} \\
\hline Молекулярная масса & \multicolumn{2}{|l|}{ 393,9 г/моль } & \multicolumn{3}{|l|}{ 1048,3 г/моль } \\
\hline Механизм действия & \multicolumn{2}{|c|}{$\begin{array}{l}\text { Взаимодействует с трансмембранным сегмен- } \\
\text { том КЧР и усиливает сигнальную трансдук- } \\
\text { цию, тем самым снижая секрецию ПтГ }\end{array}$} & \multicolumn{3}{|c|}{ Снижает секрецию ПтГ за счет активации КчР } \\
\hline Способ применения & \multicolumn{2}{|l|}{ Ежедневно внутрь } & \multicolumn{3}{|c|}{ Внутривенно в конце каждого сеанса ГД } \\
\hline \multirow{2}{*}{\multicolumn{2}{|c|}{$\begin{array}{l}\text { лируемое [10], второе - сравнительное, двойное слепое } \\
\text { рандомизированное исследование цинакальцет vs этел- } \\
\text { кальцецид [9]. } \\
\text { Цель - оценить безопасность и эффективность этел- }\end{array}$}} & \multicolumn{4}{|c|}{$\begin{array}{l}\text { Таблица 3. Обзор безопасности применения ЭКЦ в сравнении с пла- } \\
\text { цебо }\end{array}$} \\
\hline & & & Показатели & $\begin{array}{l}\text { Плацебо } \\
(\mathrm{n}=513)\end{array}$ & $\begin{array}{c}\text { ЭКЦ } \\
(n=503)\end{array}$ \\
\hline \multicolumn{2}{|c|}{$\begin{array}{l}\text { кальцетида у пациентов с ХЫІІ и неконтролируемым ВІІІІ, } \\
\text { находящихся на гемодиализе, основываясь на объединен- }\end{array}$} & \multicolumn{2}{|c|}{ Снижение уровня кальция в крови } & $10,1 \%$ & $63,8 \%$ \\
\hline \multirow{3}{*}{\multicolumn{2}{|c|}{$\begin{array}{l}\text { ных результатах двух параллельных исследований } 3 \text { фазы. } \\
\text { Независимо от группы лечения, все пациенты получали } \\
\text { стандартную терапию ВГпт, которая включает фосфат-свя- } \\
\text { зывающие препараты и кальцитриол или активные формы }\end{array}$}} & \multicolumn{2}{|c|}{ Мышечные спазмы } & $6,6 \%$ & $11,5 \%$ \\
\hline & & \multicolumn{2}{|l|}{ Диарея } & $8,6 \%$ & $10,7 \%$ \\
\hline & & \multicolumn{2}{|l|}{ Тошнота } & $6,2 \%$ & $10,7 \%$ \\
\hline \multicolumn{2}{|c|}{ витамина Д, по назначению исследователя. } & \multicolumn{2}{|l|}{ Рвота } & $5,1 \%$ & $8,9 \%$ \\
\hline \multirow{2}{*}{\multicolumn{2}{|c|}{$\begin{array}{l}\text { Критериями включения в исследования являлись: } \\
\text { взрослые пациенты } \geq 18 \text { лет, получающие гемодиализ } \\
3 \text { р/нед. в течение } \geq 3 \text { месяцев; ПтГ > } 500 \text { пг/мл и уровень }\end{array}$}} & \multicolumn{2}{|c|}{ Головная боль } & $6,0 \%$ & $7,6 \%$ \\
\hline & & \multicolumn{2}{|c|}{ Симптоматическая гипокальциемия } & $0,2 \%$ & $7,0 \%$ \\
\hline \multicolumn{2}{|c|}{$\begin{array}{l}\text { альбумин-корригированного кальция (кСа) } \geq 8,3 \text { мг/мл } \\
\text { по результатам двух последовательных измерений в раз- }\end{array}$} & \multicolumn{2}{|c|}{$\begin{array}{l}\text { Гиперкалиемия/повышенный уро- } \\
\text { вень калия }\end{array}$} & $3,1 \%$ & $4,4 \%$ \\
\hline \multicolumn{2}{|c|}{$\begin{array}{l}\text { ные дни в течение 2-х недель до рандомизации; отсутствие } \\
\text { значительного изменения дозы препаратов кальция, фос- }\end{array}$} & \multicolumn{2}{|l|}{ Смерть } & $2,9 \%$ & $2,2 \%$ \\
\hline
\end{tabular}
фат-связывающих препаратов, кальция в диализате или активной формы витамина Д в течение 4-х недель до скрининга.

В плацебо-контролируемое исследование было включено 1023 пациента: 509 получали ЭКЦ, 514 - плацебо. Пациенты получали ЭКЦ или плацебо + фосфат-связывающие препараты и кальцитриол или активные формы витамина Д, если они были назначены исследователями. Начальная доза этелкальцетида или плацебо составляла 5 мг и могла быть увеличена, с приростом 2,5 или 5 мг через 5, 9, 13 и 17 недель до максимальной дозы 15 мг. Длительность лечения - 27 недель.

Конечные точки исследований: первая - доля пациентов со снижением среднего уровня ПТГ >30\% во время периода оценки (недели 20-27). Вторая - доля пациентов со средним уровнем ПТГ $\leq 300$ пг/мл; относительное снижение ПТГ, кСа, кСа х фосфат и фосфата.

В результате исследования в группе этелкальцетида достигли снижения исходного среднего уровня ПТГ >30\% $74,7 \%$ пациентов, а в группе плацебо - 8,9\% ( $<<0,001)$. Что касается второй конечной точки: у 51,5 \% пациентов в группе ЭКЦ птГ снизился менее 300 нг/мл, а в группе плацебо - только у 4,9\% ( $<<0,001)$.

Лечение ЭКЦ ассоциировалось с большим снижением уровня кальция, фосфатов и FGF23, костной щелочной фосфатазы (КЩФ) и С-концевого телопептида коллагена 1 типа (СТх) в сравнении с плацебо.

В табл. 2 представлены данные по нежелательным явлениям (НЯ).

Как видно из табл. 2, НЯ наблюдались у 92\% и 80\% пациентов, получавших этелкальцетид и плацебо соответствен-

но. Наиболее частыми НЯ в группе этелкальцетида были тошнота, рвота, снижение уровня кальция в крови и диарея. Симптомы, потенциально связанные с гипокальциемией, включали мышечные спазмы, головную боль и парестезии. Значимое, но клинически бессимптомное удлинение интервала QT наблюдалось у некоторых пациентов в группе этелкальцетида, которое могло быть связано с изменением уровня кальция. Гиперкалиемия чаще описывалась в группе этелкальцетида; подробный анализ этих событий не выявил достоверных факторов риска или ассоциированных НЯ. Частота смертельного исхода, подтвержденных серьезных нефатальных сердечнососудистых НЯ и судорог была сопоставима в обеих группах лечения.

Таким образом, проведенное исследование показало высокую эффективность ЭКЦ в сравнении с плацебо у пациентов с умеренным и тяжелым ВГПТ, получающих гемодиализ, проанализированную по всем основным и дополнительным конечным точкам, также показана безопасность применения и удовлетворительная переносимость.

Другое крупное многоцентровое международное исследование [9] было посвящено изучению сравнительной эффективности и безопасности двух кальцимиметиков: внутривенного ЭКЦ, который вводится 3 раза в неделю во время гемодиализа, предполагая высокую комплаентность, и цинакальцета, принимаемого внутрь ежедневно в домашних условиях.

- Гипотеза исследования: эффективность этелкальцетида при ВГПТ не уступает цинакальцету по количеству пациентов со снижением уровня додиализной концентрации ПТГ >30\% во время периода оценки эффективности 
- Цель: сравнить эффективность и безопасность пероральной формы цинакальцета с внутривенным этелкальцетидом у пациентов с ВГПТ, находящихся на гемодиализе

- Дизайн: рандомизированное двойное слепое исследование с двойной маскировкой и активным контролем 3 фазы.

Рандомизировано 683 пациента в 2 группы: ЭКЦ 3 р/нед в/в + плацебо ежедневно внутрь $(n=340)$, начальная доза этелкальцетида составляла 5 мг и могла быть увеличена через 5, 9, 13 и 17 недель до максимальной дозы 15 мг; ЦКТ ежедневно внутрь + Плацебо 3 р/нед в/в ( $n=343)$, начальная доза цинакальцета составляла 30 мг и могла быть увеличена через 5, 9, 13 и 17 недель до максимальной дозы 180 мг. Длительность лечения - 26 недель (16 недель титрация дозы, 10 недель поддержание дозы), 30 дней наблюдение.

Критерии включения: сывороточные додиализные концентрации ПТГ> 500 пг/мл и альбумин-кСа $\geq 8,3$ мг/дл по результатам исследования в центральной лаборатории на этапе скрининга (реально по исходным характеристикам средний уровень ПТГ в группе ЭКЦ составил - 1092 пг/мл, а в группе ЦКТ - 1139 пг/мл, соответственно медиана 900 и 930 пг/мл); отсутствие терапии цинакальцетом в течение 3-х месяцев; концентрация кальция в диализате $\geq 2,5$ мкэв/л не менее чем за 4 недели до скрининга; стабильная доза кальцитриола, альфакальцидола или парикальцитола в течение 4-х недель, препаратов кальция - в течение 2-х недель и фосфат-связывающих препаратов - в течение 2-х недель до скрининга.

Первичная конечная точка: снижение средней исходной додиализной концентрации ПТГ >30\% во время периода оценки эффективности (не меньшая эффективность)

Основные вторичные конечные точки: снижение средней исходной додиализной концентрации ПТГ >50\% во время периода оценки эффективности (преимущество); снижение средней исходной додиализной концентрации ПТГ > 30\% за это же время (преимущество). Среднее количество дней в неделю с эпизодами тошноты или рвоты в течение первых 8 недель

Другие вторичные точки: относительное изменение средней исходной сывороточной додиализной концентрации сСа во время периода оценки эффективности: достигнута средней сывороточная додиализная концентрация P $\leq 4,5$ мг/дл. Средняя степень тяжести тошноты в течение первых 8 недель, среднее число эпизодов рвоты в неделю в течение первых 8 недель

Таблица 4. Результаты по другим вторичным точкам

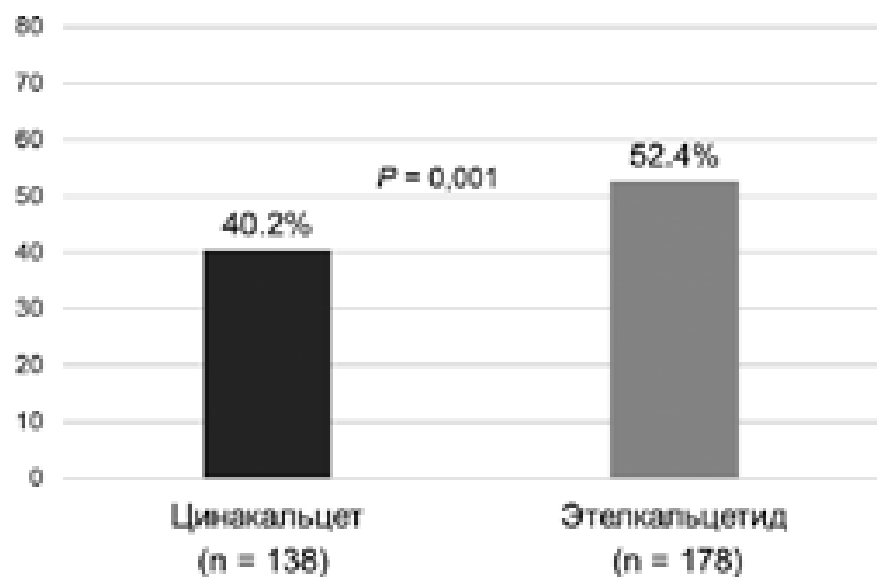

Рис. 1. Доля пациентов со снижением ПтГ более, чем на 50\%.

Поисковые вторичные точки: изменение исходных сывороточных концентраций КЩФ, FGF-23 и СТХ через 27 недель

Конечные точки безопасности: частота снижения сСа менее 7,5, 8,0 или 8,3 мг/дл в любой момент времени в ходе исследования, симптоматическая гипокальциемия, характер, частота, тяжесть и отношение к нежелательным явлениям (НЯ).

В результате проведенного исследования были получены следующие данные:

- ЭКЦ не уступал по эффективности ЦКТ в по доле пациентов, у которых ПТГ снизился более, чем на 30\% (77,9\% для ЭКЦ и 63,9\% для ЦКТ соответственно).

- ЭКЦ превосходил эффект ЦКТ по доле пациентов, у которых ПТГ снизился в период оценки эффективности более, чем на 50\% (соответственно 52 и 40\%, р <0,001) рис. 1

- Лечение ЭКЦ ассоциировалось с большим снижением сСа, чем при приеме ЦКТ: эпизоды гипокальциемии наблюдались у 68,9\% пациентов из группы ЭКЦ и у 59,8\% из группы ЦКТ, в обеих группах была потребность в увеличении дозы или назначении кальцийсодержащих препаратов и/или активных метаболитов витамина D.

В таблице 4 суммированы результаты по другим конечным точкам. Как видно из таблицы, более значимо уровень кальция снижался в группе ЭКЦ, различий по динамике фосфора, частоте эпизодов тошноты и рвоты не было.

В этом исследовании также оценивалось влияние обоих препаратов на костные маркеры и ФРФ23. Было продемонстрировано значимо большее снижение указанных показателей в группе ЭКЦ: костной щелочной фосфатазы - на 29\%, С-терминального телопептида - на 36\% и ФРФ23 - на 68\%. В группе ЦКТ эти цифры составляли 12\%, 14\% и 41\% соответственно. Авторы придают большое

\begin{tabular}{|c|c|c|c|}
\hline Показатели & $\begin{array}{l}\text { Цинакальцет } \\
(\mathrm{n}=343) \%\end{array}$ & $\begin{array}{l}\text { Этелкальцетид } \\
(\mathrm{n}=340) \%\end{array}$ & $\begin{array}{l}\text { Номинальное } \\
\text { р-значение }\end{array}$ \\
\hline $\begin{array}{l}\text { Относительное изменение исходной средней концентрации } \\
\text { кСа во время ПОЭ, среднее значение (SE) }\end{array}$ & $-6,28(0,44)$ & $-9,83(0,49)$ & $<0,001$ \\
\hline $\begin{array}{l}\text { Доля пациентов со средней концентрацией P } \leq 4,5 \text { мг/дл } \\
\text { во время ПОЭ, \% }\end{array}$ & 29,2 & 32,1 & 0,41 \\
\hline $\begin{array}{l}\text { Средняя степень тяжести тошноты в течение первых } 8 \text { не- } \\
\text { дель, скорректированное среднее значение (SE) }\end{array}$ & $0,48(0,06)$ & $0,45(0,06)$ & 0,71 \\
\hline $\begin{array}{l}\text { Среднее число эпизодов рвоты в неделю в течение первых } \\
8 \text { недель, скорректированное среднее значение (SE) }\end{array}$ & $0,1(0,02)$ & $0,2(0,02)$ & 0,26 \\
\hline
\end{tabular}


значение снижению ФРФ23. Имеются данные, что с более выраженным повышением ФРФ23 ассоциируется развитие гипертрофии левого желудочка и сердечной недостаточности у пациентов на гемодиализе $[28,29]$. В исследовании EVOLVE [30], изучавшим долгосрочный эффект лечения ЦКТ при ВГПТ с оценкой клинических исходов, В том числе сердечно-сосудистых событий, было показано, что 30\% снижение ФРФ23 к 20 неделям лечения ассоциировалось со значимым снижением частоты сердечной недостаточности и внезапной смерти.

С другой стороны, в сравнительном исследовании ЭКЦ и ЦКТ наблюдался количественный дисбаланс для НЯ сердечной недостаточности в группе этелкальцетида (10 [3,0\%]) по сравнению с группой цинакальцета (2 [0,6\%]). НЯ сердечной недостаточности были серьезными у 5 (в том числе 3 фатальных НЯ) и 1 пациента (нефатальные НЯ) в группах этелкальцетида и цинаальцета соответственно. Не описывалось ни одного случая фатального или серьезного НЯ сердечной недостаточности на фоне гипокальциемии; 2 несерьезных НЯ в группе этелкальцетида были связаны с низкими показателями сСа - 8,0 и 8,1 мг/дл. Ни одно НЯ не оценивалось исследователем как связанное с исследуемым препаратом

Таким образом проведенное сравнительное исследование эффективности и безопасности применения ЭКЦ и ЦКТ при ВГПТ позволяет сделать следующие выводы:

- Этелкальцетид позволил достичь снижения концентрации ПтГ > 50\% и > 30\% у большего количества пациентов по сравнению с цинакальцетом, при этом частота развития тошноты и рвоты не отличалась между группами

- Наблюдался количественный дисбаланс для НЯ сердечной недостаточности, однако причинно-следственная связь с этелкалцетидом не может быть установлена

- Гипокальциемия чаще описывалась в группе этелкальцетида

- Внутривенное введение этелкальцетида эффективнее перорального цинакалцета для лечения пациентов с ВГПТ, находящихся на ГД

- Можно предположить повышение эффективности лечения за счет лучшей приверженности к лечению за счет меньшей кратности и способа введения ЭКЦ в сравнении с ЦКТ

Необходимо отметить ограничения этого исследования: его краткосрочность (26 недель), оценку эффективности по суррогатным критериям, а не конечным клиническим исходам, ограниченность диализной популяции по длительности диализа (в среднем 4 года) и возрасту (средний возраст 55 лет).

Тем не менее очень важным представляется способ введения ЭКЦ внутривенно 3 раза в неделю во время диализа.
Ряд исследований продемонстрировал низкую приверженность диализных больных к соблюдению диетического режима, потреблению жидкости и приему пероральных препаратов в ежедневном режим, что может быть связано с необходимостью большого приема таблеток «от давления», снижающих холестерин, препаратов витамина Д, кальция, севеламера и, наконец, ЦКТ. Каждый таблетированный препарат способен вызывать те или иные нежелательные явления, а их комбинация чревата большей частотой осложнений со стороны ЖКТ. [31]. В исследовании посвященном приверженности к лечению ЦКТ диализных больных с ВГПТ было показано (включено 4923 пациента), что после 12 месяцев наблюдения, только 28\% пациентов были полностью комплаенты и длительно продолжали лечение; 46\% - прекратили лечение, и 23\% были частично привержены к лечению. Лучшая приверженность к лечению ассоциировалась со значительно меньшими затратами на лечение госпитализированных пациентов (8899\$ vs 5858\$) в год[32]

\section{ЗАКЛЮЧЕНИЕ}

Лечение ВГПТ при терминальной ХБП представляет серьезную проблему, особенно при тяжелых формах ВГВТ, что связано с необходимостью многокомпонентной терапии, частым развитием побочных эффектов, возникновением резистентности к медикаментозной терапии. Важным патогенгетическим звеном комплексного лечения ВГПТ является применение агонистов КЧР - кальцимиметиков. Разработка кальцимиметика для внутривенного введения - новый этап в усовершенствовании лечения ВГПТ и повышения его эффективности.

Данные опубликованных исследований свидетельствуют о большей эффективности этелкальцетида в сравнении с цинокальцетом. цинакальцета. При этом, режим дозирования этелкальцетида и возможность его введения в диализном центре могут обеспечить 100 комплантность пациентов и уменьшить сложности с получением препарата.

Несомненно, необходимы дальнейшие клинические исследования, связывающие улучшение биохимических показателей с клиническими исходами [33]: увеличит ли этелкальцетид длительность жизни больных и каково его влияние на качество жизни; возможны ли комбинации препаратов, повышающие эффективность этелкальцетида.

\section{ДОПОЛНИТЕЛЬНАЯ ИНФОРМАЦИЯ}

Конфликт интересов. Авторы декларируют отсутствие явных и потенциальных конфликтов интересов, связанных с публикацией настоящей статьи

\section{СПИСОК ЛИТЕРАТУРЫ | REFERENCES}

1. Егшатян Л.В., Рожинская Л.Я. Медикаментозные методы коррекции почечной остеодистрофии // Остеопороз и остеопатии. 2014. - T. 17. - №2. - C. 29-35. [Egshatyan LV, Rozhinskaya LY. Medical methods of correction of renal osteodystrophy. Osteoporosis and Bone Diseases. 2014;17(2):29-35. (In Russ.)] doi: 10.14341/osteo2014229-35.

2. Collins AJ, Kasiske B, Herzog C, et al. Excerpts from the United States Renal Data System 2004 Annual Data Report: Atlas of end-stage renal disease in the United States. Am J Kidney Dis. 2005;45:A5-A7. doi: 10.1053/j.ajkd.2004.10.009.
3. Coresh J, Astor BC, Greene T, et al. Prevalence of chronic kidney disease and decreased kidney function in the adult US population: Third national health and nutrition examination survey. Am J Kidney Dis. 2003:41(1):1-12. doi: 10.1053/ajkd.2003.50007.

4. Levin A., Stevens PE., Bilous RW. et al. Kidney disease: Improving global outcomes (KDIGO) CKD work group. KDIGO 2012 clinical practice guideline for the evaluation and management of chronic kidney disease. Kidney International Supplements. 2013;3(1):1-150. doi: 10.1038/kisup.2012.73 
5. Гребенникова Т.А. Белая Ж.Е., Цориев ТТ, и др. Эндокринная функция костной ткани // Остеопороз и остеопатии. - 2015. T. 18. - №1 - - C. 28-37. [Grebennikova TA, Belaya ZE, Tsoriev TT, et al. The endocrine function of the bone tissue. Osteoporosis and Bone Diseases. 2015;18(1):28-37. (In Russ.)] doi: 10.14341/osteo2015128-37.

6. Fukumoto S, Yamashita T. FGF23 is a hormone-regulating phosphate metabolism - Unique biological characteristics of FGF23. Bone. 2007;40(5):1190-1195. doi: 10.1016/j.bone.2006.12.062.

7. Martin A, Quarles LD. Evidence for FGF23 Involvement in a Bone-Kidney Axis Regulating Bone Mineralization and Systemic Phosphate and Vitamin D Homeostasis. 2012;728:65-83. doi: 10.1007/978-1-4614-0887-1_4

8. Jean G, Terrat JC, Vanel T, et al. High levels of serum fibroblast growth factor (FGF)-23 are associated with increased mortality in long haemodialysis patients. Nephrology Dialysis Transplantation. 2009;24(9):2792-2796. doi: 10.1093/ndt/gfp191.

9. Block GA, Bushinsky DA, Cheng $S$, et al. Effect of Etelcalcetide vs Cinacalcet on Serum Parathyroid Hormone in Patients Receiving Hemodialysis With Secondary Hyperparathyroidism. JAMA. 2017;317(2):156. doi: 10.1001/jama.2016.19468.

10. Block GA, Bushinsky DA, Cunningham J, et al. Effect of Etelcalcetide vs Placebo on Serum Parathyroid Hormone in Patients Receiving Hemodialysis With Secondary Hyperparathyroidism. JAMA. 2017;317(2):146. doi: 10.1001/jama.2016.19456.

11. Brown EM, Gamba G, Riccardi D, et al. Cloning and characterization of an extracellular Ca2+-sensing receptor from bovine parathyroid. Nature. 1993;366(6455):575-580. doi: 10.1038/366575a0.

12. Conigrave AD, Quinn SJ, Brown EM. Cooperative multi-modal sensing and therapeutic implications of the extracellular $\mathrm{Ca} 2+$ sensing receptor. Trends Pharmacol Sci. 2000;21(10):401-407. doi: 10.1016/s0165-6147(00)01546-7.

13. Block GA, Martin KJ, de Francisco ALM, et al. Cinacalcet for Secondary Hyperparathyroidism in Patients Receiving Hemodialysis. N Eng/J Med. 2004;350(15):1516-1525. doi: 10.1056/NEJMoa031633.

14. Ермоленко В.М., Ветчинникова ОН, Волгина ГВ, и др. Минеральные и костные нарушения при хронической болезни почек (МКН-ХБП) - клинические рекомендации Научного общества нефрологов России. - 2015. EErmolenko VM, Vetchinnikova ON, Volgina GV, et al. Mineral'nye i kostnye narushenija pri hronicheskoj bolezni pochek (MKN-HBP);2015 (In Russ.)]

15. Nemeth EF. Pharmacodynamics of the Type II Calcimimetic Compound Cinacalcet HCl. J Pharmacol Exp Ther. 2003;308(2):627-635. doi: 10.1124/jpet.103.057273.

16. Moe SM, Chertow GM, Coburn JW, et al. Achieving NKF-K/ $\mathrm{DOQI}^{\mathrm{TM}}$ bone metabolism and disease treatment goals with cinacalcet HCl. Kidney Int. 2005;67(2):760-771. doi: 10.1111/j.1523-1755.2005.67139.x.

17. Malluche HH, Monier-Faugere MC, Wang G, et al. An assessment of cinacalcet $\mathrm{HCl}$ effects on bone histology in dialysis patients with secondary hyperparathyroidism. Clin Nephrol. 2008;69(04):269-278. doi: 10.5414/cnp69269.

18. Sprague SM, Wetmore JB, Gurevich K, et al. Effect of Cinacalcet and Vitamin D Analogs on Fibroblast Growth Factor-23 during the Treatment of Secondary Hyperparathyroidism. Clin J Am Soc Nephrol. 2015;10(6):1021-1030. doi: 10.2215/cjn.03270314.
19. Cunningham J, Danese $\mathrm{M}$, Olson $\mathrm{K}$, et al. Effects of the calcimimetic cinacalcet $\mathrm{HCl}$ on cardiovascular disease, fracture, and health-related quality of life in secondary hyperparathyroidism. Kidney Int. 2005;68(4):1793-1800. doi: 10.1111/j.1523-1755.2005.00596.x.

20. Moe SM, Abdalla S, Chertow GM, et al. Effects of Cinacalcet on Fracture Events in Patients Receiving Hemodialysis: The EVOLVE Trial. J Am Soc Nephrol. 2014;26(6):1466-1475. doi: 10.1681/asn.2014040414.

21. Padhi D, Harris R. Clinical Pharmacokinetic and Pharmacodynamic Profile of Cinacalcet Hydrochloride. Clin Pharmacokinet. 2009;48(5):303-311. doi: 10.2165/00003088-200948050-00002.

22. Gincherman Y, Moloney K, McKee C, Coyne DW. Assessment of adherence to cinacalcet by prescription refill rates in hemodialysis patients. Hemodialysis International. 2010;14(1):68-72. doi: 10.1111/j.1542-4758.2009.00397.x.

23. Walter S, Baruch A, Dong J, et al. Pharmacology of AMG 416 (Velcalcetide), a Novel Peptide Agonist of the Calcium-Sensing Receptor, for the Treatment of Secondary Hyperparathyroidism in Hemodialysis Patients. J Pharmacol Exp Ther. 2013;346(2):229-240. doi: 10.1124/jpet.113.204834

24. Goodman WG. Calcimimetic agents and secondary hyperparathyroidism: treatment and prevention. Nephrology Dialysis Transplantation. 2002;17(2):204-207. doi: 10.1093/ndt/17.2.204.

25. Chen P, Melhem M, Xiao J, et al. Population pharmacokinetics analysis of AMG 416, an allosteric activator of the calcium-sensing receptor, in subjects with secondary hyperparathyroidism receiving hemodialysis. The Journal of Clinical Pharmacology. 2015;55(6):620-628. doi: 10.1002/jcph.460.

26. Goodman WG, Quarles LD. Development and progression of secondary hyperparathyroidism in chronic kidney disease: lessons from molecular genetics. Kidney Int. 2008;74(3):276-288. doi: 10.1038/sj.ki.5002287.

27. Fukagawa M, Yokoyama K, Shigematsu T, et al. A phase 3, multicentre, randomized, double-blind, placebo-controlled, parallel-group study to evaluate the efficacy and safety of etelcalcetide (ONO-5163/AMG 416), a novel intravenous calcimimetic, for secondary hyperparathyroidism in Japanese haemodialysis patients. Nephrology Dialysis Transplantation. 2017:gfw408. doi: 10.1093/ndt/gfw408.

28. Faul C, Amaral AP, Oskouei B, et al. FGF23 induces left ventricular hypertrophy. J Clin Invest. 201 1;121(11):4393-4408. doi: 10.1172/jci46122.

29. Poelzl G, Trenkler C, Kliebhan J, et al. FGF23 is associated with disease severity and prognosis in chronic heart failure. Eur J Clin Invest. 2014;44(12):1150-1158. doi: 10.1111/eci.12349.

30. Chertow GM, Pupim LB, Block GA, et al. Evaluation of Cinacalcet Therapy to Lower Cardiovascular Events (EVOLVE): Rationale and Design Overview. Clin J Am Soc Nephrol. 2007;2(5):898-905. doi: 10.2215/cjn.04381206.

31. Clark S, Farrington $\mathrm{K}$, Chilcot J. Nonadherence in Dialysis Patients: Prevalence, Measurement, Outcome, and Psychological Determinants. Seminars in Dialysis. 2014;27(1):42-49. doi: 10.1111/sdi.12159.

32. Lee A, Song $X$, Khan I, et al. Association of cinacalcet adherence and costs in patients on dialysis. J Med Econ. 2011;14(6):798-804. doi: 10.3111/13696998.2011.627404.

33. Middleton JP, Wolf M. Second Chances to Improve ESRD Outcomes With a Second-Generation Calcimimetic. JAMA. 2017;317(2):139. doi: 10.1001/jama.2016.18631.

\section{ИНФОРМАЦИЯ ОБ АВТОРАХ [AUTHORS INFO]}

Рожинская Людмила Яковлевна, Д.М.Н., профессор [Liudmila Y. Rozhinskaya, MD, PhD, professor]; aдpec: 117036, г. Москва, ул. Дм. Ульянова, д.11 [address: 117036, Russia, Moscow, Dmitria Uljanova street, 11]; ORCID: http://orcid.org/0000-0001-7041-0732; eLibrary SPIN: 5691-7775; e-mail: rozhinskaya@rambler.ru Белая Жанна Евгеньевна, доктор медицинских наук [Zhanna E. Belaya, MD, PhD]; e-mail: jannabelaya@gmail.com; ORCID: http://orcid.org/0000-0002-66746441; eLibrary SPIN: 4746-7173.

Луценко Александр Сергеевич, [Alexander S. Lutsenko, MD]; e-mail: some91@mail.ru; ORCID: http://orcid.org/0000-0002-9314-7831; eLibrary SPIN: 4037-1030.

\section{цИТИРОВАТЬ:}

Рожинская Л.Я., Белая Ж.Е., Луценко А.С. Новые возможности лечения вторичного гиперпаратиреоза у пациентов с терминальной стадией хронической болезни почек, получающих заместительную почечную терапию гемодиализом. // Остеопороз и остеопатии. — 2017. — Т. 20. — №1. — C.32-38. doi: 10.14341/osteo2017126-33

\section{TO CITE THIS ARTICLE:}

Rozhinskaya LY, Belaya ZE, Lutsenko AS. Novel treatment options for secondary hyperparathyroidism in end-stage kidney disease patients on hemodialysis therapy. Osteoporosis and bone diseases. 2017;20(1):32-38. doi: 10.14341/osteo2017126-33 